\title{
Dietary CDP-choline supplementation prevents memory impairment caused by impoverished environmental conditions in rats
}

\author{
Lisa A. Teather ${ }^{1,2}$ and Richard J. Wurtman \\ Department of Brain and Cognitive Sciences, Massachusetts Institute of Technology, Cambridge, Massachusetts 02139, USA
}

\begin{abstract}
We previously showed that dietary cytidine (5')-diphosphocholine (CDP-choline) supplementation could protect against the development of memory deficits in aging rats. In the present study, younger rats exposed to impoverished environmental conditions and manifesting hippocampal-dependent memory impairments similar to those observed in the aging rodents were given CDP-choline, and its effects on this cognitive deficit were assessed. Male Sprague-Dawley rats reared for $3 \mathrm{mo}$ in impoverished (IC) or enriched environmental (EC) conditions concurrently received either a control diet or a diet supplemented with CDP-choline $(\sim 500 \mathrm{mg} / \mathrm{kg} / \mathrm{d})$. After $3 \mathrm{mo}$, rats were trained to perform spatial and cued versions of the Morris water maze, and their rates of acquisition and retention were compared. Impoverished rats exhibited a selective deficit in hippocampal-dependent spatial memory which could be ameliorated by feeding them CDP-choline. The CDP-choline had no memory-enhancing effect in enriched rats, nor did it prevent the memory impairment of impoverished rats if the animals consumed it for the initial or final months instead of for the entire 3-mo period. These findings indicate that long-term dietary CDP-choline supplementation can ameliorate the hippocampal-dependent memory impairment caused by impoverished environmental conditions in rats, and suggest that its actions result, in part, from a long-term effect such as enhanced membrane phosphatide synthesis, an effect shown to require long-term dietary supplementation with CDP-choline.
\end{abstract}

The environmental conditions under which rats are reared can affect subsequent cognitive performance (Hebb 1949), especially forms of cognition that depend on the hippocampus (e.g., Juraska and Muller 1984; Kempermann et al. 1997; Duffy et al. 2001; Teather et al. 2002). Interestingly, advanced age is associated with a similar decline in hippocampal-dependent memory capacities in humans (e.g., Craik 1990; Albert 1993) and certain animals (e.g., Barnes et al. 1980; Gage et al. 1988; Gallagher et al. 1993). In rats, both aging (e.g., Rapp et al. 1987; Gallagher and Pelleymounter 1988; Teather and Wurtman 2003) and impoverished conditions (Teather et al. 2002) result in spatial learning and memory deficits. Hippocampal function is requisite for proper spatial memory processing (Morris et al. 1982; Packard and Teather 1997, 1998). Neither aging (Teather et al. 2002) nor impoverished environmental conditions (Teather and Wurtman 2003) compromise procedural memory, perhaps because procedural memory (also known as habit or stimulus-response memory) is independent of hippocampal function (Packard et al. 1989; Packard and Teather 1997, 1998).

In a recent study we observed that, compared with young rats, aging rats (i.e., 18-mo-old) had difficulty mastering a hippocampal-dependent spatial task, and that long-term dietary supplementation with (5')-diphosphocholine (CDP-choline) prevented this memory impairment (Teather and Wurtman 2003). Inasmuch as the cognitive dysfunctions manifested by aged rats and rats exposed to impoverished environmental conditions are similar, it seemed possible that similar physiological mechanisms might underlie these impairments, in which case dietary supple-

\footnotetext{
1Present address: Dept. of Psychology, Wilfrid Lauer University, Waterloo, Ontario, Canada N2L 3C5.

${ }^{2}$ Corresponding author.

E-mail Iteather@mit.edu; fax (519) 746-7605.

Article published online ahead of print. Article and publication date are at http://www.learnmem.org/cgi/doi/10.1101/lm.83905.
}

mentation with CDP-choline might also ameliorate the memory impairment caused by environmental impoverishment.

We thus examined the effect of CDP-choline on hippocampal-dependent and striatal-dependent forms of memory processing among rats exposed to enriched (EC) or impoverished conditions (IC) for 3 mo starting at weaning. Preliminary work in our laboratory had indicated that 3 mo of IC reliably elicits hippocampal-dependent memory deficits. Moreover, our earlier studies on aging indicated that long-term dietary CDP-choline supplementation would be needed in order to treat the cognitive impairments of such animals (Teather and Wurtman 2003). Thus, we also conducted a second experiment to determine whether treating the IC rats with the CDP-choline supplemented diet for only the initial or final month of environmental manipulation could also improve cognitive function, or whether the full $3 \mathrm{mo}$ of treatment would be necessary.

\section{Results}

\section{Body weight}

Animals were weighed weekly to ensure that treated and untreated rats were eating equivalent amounts of food. No significant differences in mean body weight were found between CDPcholine-supplemented and control groups (data not shown), indicating that rats were eating equivalent amounts of diet whether it was supplemented with CDP-choline or not. Also, as IC rats were exercised to avoid the weight gain that often occurs in these rats (relative to EC rats), there was no difference in body weight among EC or IC groups (data not shown).

\section{Experiment 1}

Two-way ANOVA analysis (diet and environment as factors) with repeated measures (days or training blocks) of the mean escape latencies across $4 \mathrm{~d}$ of training in the hidden platform task 
showed a significant main effect of day $\left[F_{(3,84)}=30.923\right.$, $P<0.001]$. All groups were able to learn the spatial task to some degree, as shown by the decrease in mean escape latency across the $4 \mathrm{~d}$ of training (Fig. 1A). ANOVA analysis also revealed significant main effects of environment $\left[F_{(1,28)}=32.61, P<0.001\right]$, $\operatorname{diet}\left[F_{(1,28)}=32.606, P<0.001\right]$, as well as a diet $\times$ environment interaction $\left[F_{(1,1)}=30.828, P<0.001\right)$. Post-hoc analysis indicated that untreated (i.e., no CDP-choline supplementation) impoverished rats had significantly higher escape latencies on all 4 $\mathrm{d}$ of training than did the other groups ( $\left.P^{\prime} \mathrm{s}<0.05\right)$.

A repeated measures ANOVA on the distance traveled to find the hidden platform also indicated a significant main effect of environment $\left[F_{(1,28)}=23.5, P<0.01\right]$ and diet $\left[F_{(1,28)}=23.76\right.$, $P<0.01]$, as well as a diet $\times$ environment interaction $\left[F_{(1,1)}=24.23, P<0.01\right)$. Post-hoc analysis indicated that untreated (i.e., no CDP-choline supplementation) impoverished rats traveled longer distances to find the hidden platform on all $4 \mathrm{~d}$ of training than did the other groups $\left(P^{\prime} \mathrm{s}<0.05\right.$, data not shown). Moreover, mean swim speed did not differ between groups (data not shown), confirming that group differences in distance traveled and escape latency were not secondary to differences in swim speed. Some rats did manifest thigmotaxic behavior on the initial trials on Day 1 of spatial training; however, thigmotaxia was not evident in any animals after trial 3 on Day 1 of training (data not shown).

Improvement across trials (and days) in the spatial water maze task is not necessarily an indication of spatial learning, because these measures do not provide information about the search strategy, and nonspatial strategies can often be used to locate the hidden platform (Gallagher et al. 1993). Thus we carried out a probe test to assess search strategy and memory for the platform's location. ANOVA analysis of the mean percentage of swim time in the four quadrants during the 60 -sec probe test revealed significant main effects of environment $\left[F_{(3,28)}=7.1\right.$,
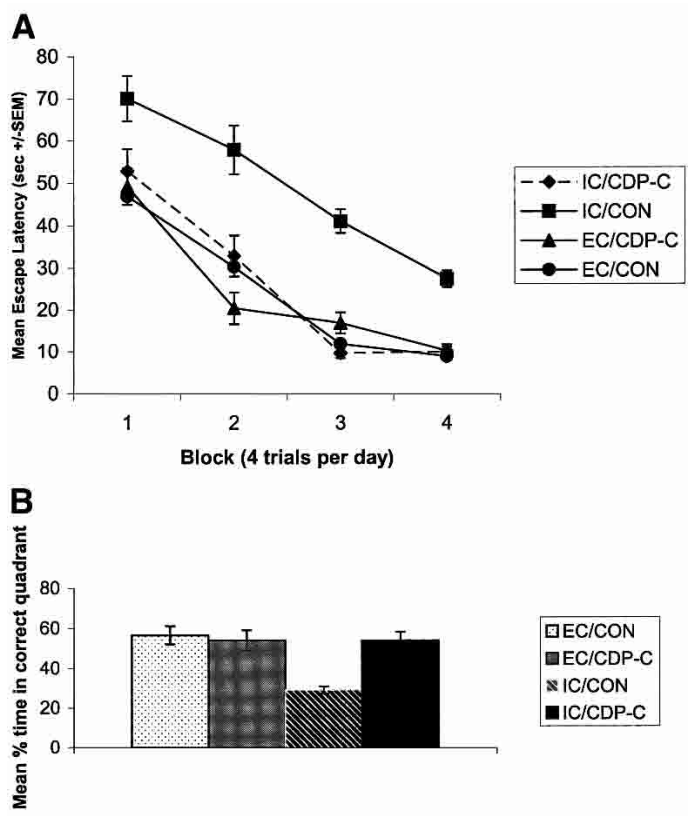

Figure 1. (A) Mean escape latencies \pm SEMs for $4 \mathrm{~d}$ of acquisition of a hidden platform water maze task. EC and IC rats were given access to either a control diet or a diet supplemented with CDP-choline $(\sim 500$ $\mathrm{mg} / \mathrm{kg} / \mathrm{d}$ ) for $3 \mathrm{mo} . n=8$ for all four experimental groups. Rats were given four training trials (i.e., block) for four consecutive days. The submerged platform was always located in the same quadrant. (B) The percent of total swim time spent by rats in the target quadrant (i.e., that previously contained the platform) during the $60-\mathrm{sec}$ probe test.

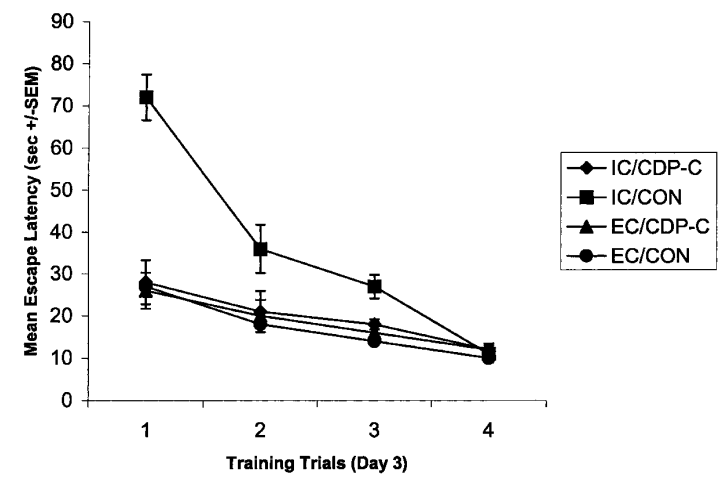

Figure 2. Untreated IC rats acquire spatial information during the spatial training sessions; however, initial escape latencies are noticeably impaired. Mean escape latencies \pm SEMs for each training trial on day 3 of acquisition of a hidden platform water maze task are shown to illustrate this effect.

$P<0.005]$ and quadrant $\left[F_{(3,28)}=8.68, P<0.001\right]$, as well as an environment $\times$ diet interaction $\left[F_{(1,1)}=4.23, P<0.01\right]$. Post-hoc analysis indicated that untreated IC rats swam for a significantly shorter time in the target quadrant than did the other groups $\left(P^{\prime} \mathrm{s}<0.05\right.$, Fig. 1B).

The escape latencies of the initial trial on each day are considered the most sensitive measure of memory for the previous day's learning (Packard and Teather 1997, 1998). When the mean escape latencies for the initial trials on the last $3 \mathrm{~d}$ of spatial training were analyzed, untreated IC rats were found to have exhibited significantly higher escape latencies than any of the other groups $\left(P^{\prime} \mathrm{s}<0.05\right)$, indicating that untreated rats displayed less memory for the previous day's learning. Figure 2 illustrates these findings from day 3 of spatial training; training days 2 and 4 displayed similar patterns (i.e., escape latency differences between the experimental groups was most predominant on trial 1 of each day). It should be noted that by trial 4 on each day, IC rats, regardless of treatment, did not differ greatly from EC rats. This indicates that the memory for the prior day's information may be poor in IC rats; IC rats can acquire the spatial task, however.

Two-way ANOVA with repeated measures analysis of the mean escape latencies across the $4 \mathrm{~d}$ of training in the visible platform task showed a significant main effect of day $\left[F_{(3,28)}=46.82, P<0.001\right]$. No main effects of diet or environment (or interactions) were revealed, indicating that all groups (regardless of environment or diet) acquired the cued water maze task at equivalent rates (Fig. 3).

\section{Experiment 2}

In previous work we had found that aged rats required at least 6 wks of dietary CDP-choline supplementation to provide cognitive benefits; short-term treatment was not effective in attenuating the age-related memory deficit (Teather and Wurtman 2003). Thus, as 3 mo of CDP-choline treatment proved to be beneficial in Experiment 1, we next explored whether long-term CDPcholine treatment would be required to alleviate the memory impairment caused by impoverished environmental conditions. Moreover, as the behavioral effects of impoverishment have been observed in as early as $1 \mathrm{mo}$, it is of interest to assess whether early (initial month of IC procedures) dietary supplementation with CDP-choline could prevent the memory impairment caused by IC, or perhaps whether late CDP-choline (last month of IC procedures) could alleviate the memory impairment caused by IC procedures. To test these possibilities, we reared four groups of rats in IC conditions. One group was fed a control diet; a second 


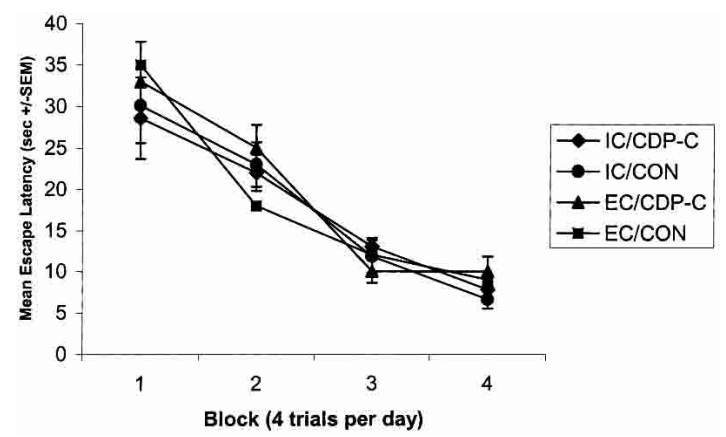

Figure 3. Mean escape latencies \pm SEMs for $3 \mathrm{~d}$ of acquisition of a visible platform water maze task. EC and IC rats given access to either a control diet or a diet supplemented with CDP-choline $(-500 \mathrm{mg} / \mathrm{kg} / \mathrm{d})$ for $3 \mathrm{mo} ; n=8$ for all four experimental groups. Rats were given four training trials (i.e., block) for three consecutive days. The visible platform was located in a different quadrant for all four training trials.

group was fed the CDP-choline diet for the entire 3 mo of IC; the third group was fed the high-CDP-choline diet for the first month of IC, then switched to the control diet for the last 2 mo of IC; the fourth group was fed the control diet for the first 2 mo of IC and the CDP-choline diet for the last month of IC.

One-way ANOVA (diet as the factor) with repeated measures (days) analysis of the mean escape latencies across the $4 \mathrm{~d}$ of training in the hidden platform task showed a significant main effect of day $\left[F_{(3,84)}=46.212, P<0.001\right]$, indicating that all groups mastered the spatial task to some extent (Fig. 4A). A significant main effect of diet was also revealed $\left[F_{(1,28)}=14.67\right.$,
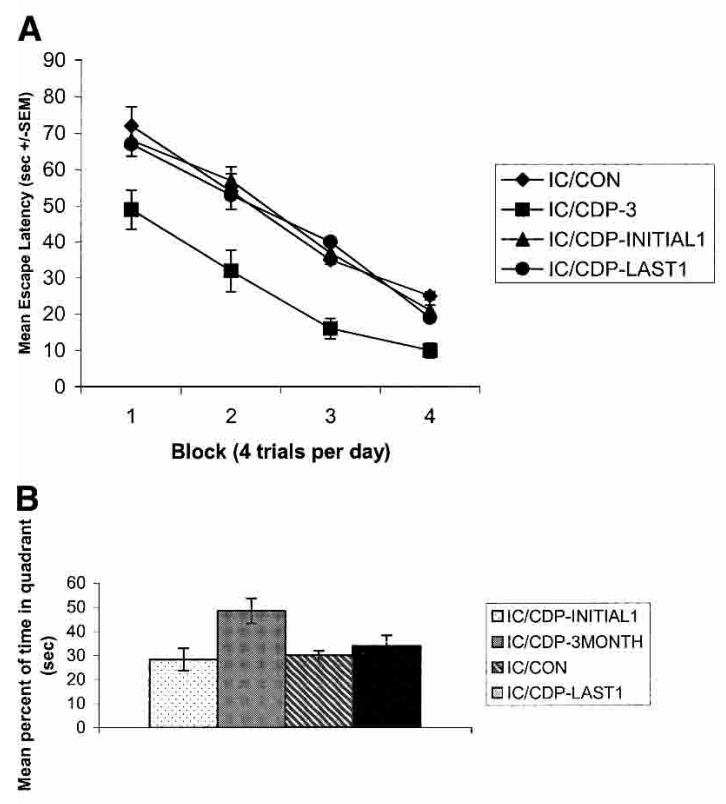

Figure 4. (A) IC rats treated with the high CDP-choline diet long-term, but not short-term, manifest superior spatial memory skills. Mean escape latencies \pm SEMs for acquisition in a hidden platform water maze task. IC rats were given access to either a control diet or a diet supplemented with CDP-choline $(-500 \mathrm{mg} / \mathrm{kg} / \mathrm{d})$ for the entire $3 \mathrm{mo}$, or for the initial month or the final month only. $n=8$ for all four experimental groups. Rats were given eight training trials with the submerged platform always located in the same quadrant. $(B)$ The percent of total swim time spent by IC rats in the target quadrant (i.e., that previously contained the platform) during the $60-\mathrm{sec}$ probe test administered $24 \mathrm{hr}$ after the initial eight training trials.
$P<0.05]$. Post-hoc analysis indicated that IC rats treated with CDP-choline for 3 mo (i.e., the entire time of IC rearing) differed significantly from untreated IC rats and IC rats treated with the high CDP-choline diet for either the initial or the final month of IC rearing procedures.

We next carried out a probe test to assess search strategy and memory for the platform's location after $4 \mathrm{~d}$ of hidden platform training. ANOVA analysis of the mean percentage of swim time in the four quadrants during the 60 -sec probe test revealed significant main effects of diet $\left[F_{(1,28)}=8.71, P<0.01\right]$ and quadrant $\left[F_{(3,28)}=9.1, P<0.01\right]$. Post-hoc analysis indicated that IC rats treated with a high CDP-choline diet for the entire 3 mo of IC procedures swam for a significantly longer time in the target quadrant than did the other groups $\left(P^{\prime} \mathrm{s}<0.05\right.$. Fig. $\left.4 \mathrm{~B}\right)$.

\section{Discussion}

These data show that rats reared under impoverished environmental conditions for 3 mo (between the ages of 30 and $120 \mathrm{~d}$ of age) display hippocampal-dependent spatial memory impairments, and that providing long-term supplemental CDP-choline via the diet can protect against development of these impairments.

The ability of untreated IC rats to acquire the spatial version of the Morris water maze was less than that of untreated IC rats, or of EC rats that did or did not receive the high CDP-choline diet (Fig. 1A). Moreover, as shown using the probe test, untreated IC rats spent less time than CDP-choline-treated IC animals, or EC rats, in the quadrant that had previously contained the platform (Fig. 1B). Inasmuch as the search strategy is acquired through prior experience, the cognitive deficit in untreated IC rats could result from failure to remember the platform's location because of inadequate consolidation of this information, or impaired retrieval mechanisms during task acquisition. It should be noted that untreated IC rats are able to acquire spatial tasks; their memory deficit may thus result from impaired transfer or consolidation of the information acquired during previous learning. Support for this hypothesis is shown by the finding that the mean retention latencies on the last trials of all $4 \mathrm{~d}$ of spatial training did not differ significantly among the groups (e.g., Fig. 2).

Interestingly, CDP-choline had no beneficial effect on enriched rats; this suggests that enriched rats may display a ceiling effect in the sense that improvement cannot be observed due to high-functioning mnemonic skills displayed by enriched rats. IC rats apparently acquire procedural memory (i.e., cued task) at a rate comparable to that of EC rats (Fig. 3). Hence their memory deficit does not appear to result from non-mnemonic effects such as impaired motivation or diminished sensorimotor skills.

Interestingly, although aging and rearing under impoverished conditions have similar negative effects on hippocampaldependent memory, neither compromises striatal-dependent memory. While the physiological bases for the memory impairments caused by aging and impoverishment have not yet been identified, the fact that supplemental CDP-choline benefits both impaired populations suggests that aging and impoverishment may have similar cellular and molecular alterations. Thus, this brings up the possibility that IC rats could serve as a useful model for studying the physiological basis of aging.

The biochemical mechanisms that underlie the ability of CDP-choline to protect against memory impairments caused by IC or aging await elucidation. However, the fact that a full 3 mo of treatment are needed for both effects (Fig. 3; Teather and Wurtman 2003) suggests that the mechanisms involve more than a rapid increase in acetylcholine synthesis and release caused by liberation of free choline in CDP-choline (Cohen and 
Wurtman 1976; Hirsh et al. 1978), which can occur immediately upon CDP-choline intake. Rather, we propose that it may be an increase in brain phosphatides that is responsible for the beneficial mnemonic effects of long-term CDP-choline. In support of this hypothesis, chronic oral administration of CDP-choline to rats for $42 \mathrm{~d}$ or longer was shown to increase brain phosphatide levels by enhancing their synthesis (Lopez-Coviella et al. 1987, 1992).

Oral CDP-choline is rapidly and completely metabolized, in rats, to cytidine and choline. These compounds readily enter the brain and are phosphorylated to cytidyl triphosphate (CTP) and phosphocholine, respectively. These intermediates can then combine to form endogenous CDP-choline, which reacts with diacylglycerol (DAG) to form phosphatidylcholine (PC). An increase in PC synthesis is rapidly followed by proportionate increases in the other membrane phosphatides (phosphoethanalomine and phosphatidylserine, and possibly in membrane proteins, so that membrane composition, and presumably, membrane function are maintained. The phosphatides are highly localized within, and indeed are the major constituent of, cellular membranes. An increase in their level, per brain cell, could affect the size of synapses, and would also increase brain reservoirs for biologically active phosphatide constituents like choline; the second messengers DAG and inositol triphosphate; phosphatidic acid; and such lipid mediators as platelet-activating factor (PAF) and arachidonic acid, the essential precursor for the prostaglandins. Virtually all of these compounds have been implicated in hippocampal-dependent memory processing in rats (e.g., Holscher et al. 1995; Moriyama et al. 1996; Teather et al. 1998, 2002).

We suggest, based on these known effects of CDP-choline, that it may alleviate the memory deficits caused by impoverished conditions by affecting synaptic size and/or post-synaptic signal transduction components, rather than simply by increasing presynaptic acetylcholine release.

\section{Materials and Methods}

\section{Animals and diet}

All animals were maintained under standard environmental conditions (room temperature, $20^{\circ}-20^{\circ} \mathrm{C}$; relative humidity, $55 \%-$ $60 \%$; light/dark schedule, $12 / 12 \mathrm{hr}$ ). Eight pregnant Sprague Dawley rats (Charles River Laboratories) were obtained 1 wk prior to giving birth. At postnatal (PN) day 23, male pups were removed and separated into small groups and allowed to acclimatize for $1 \mathrm{wk}$. At this time, pups were assigned to either the EC or IC condition; rats were matched according to body weight at PN day 23. One subgroup of IC rats $(n=8)$ and one subgroup of EC rats $(n=8)$ were given access to a diet of lab chow supplemented with CDP-choline (generously provided by Ferrer Internacional). The remaining subgroups $(n ' s=8)$ received the unsupplemented control diet (Teklad Global 16\% protein rodent diet; Harlen Teklad). Thus, the rats were fed the diets and were enriched or impoverished from 30 to $120 \mathrm{~d}$ of age.

To assess whether CDP-choline supplementation could prevent memory impairments in IC rats when administered either early or late in rearing, a second experiment was carried out. In this second experiment four groups of IC rats were used. In preliminary studies, we found that CDP-choline treatment did ameliorate memory impairments in IC rats, yet had no effect on EC rat performance (L. Teather and R. Wurtman, unpubl.); therefore we elected to conserve animal usage by using only IC rats. One IC group received the high CDP-choline diet for the first month of rearing, followed by control diet for the final $2 \mathrm{mo}$; another IC group received the control diet for the initial 2 mo of rearing, followed by the high CDP-choline diet for the last month of rearing. Another IC group received the high CDP-choline diet for the entire $3 \mathrm{mo}$, and the final IC group received a control diet for the entire 3 mo.
Four diets were prepared containing different amounts of CDP-choline. Mean weekly intakes were monitored for all rats, and the diets were adjusted so that rats would receive $\sim 500 \mathrm{mg} /$ $\mathrm{kg} / \mathrm{d}$. It should be noted that EC rats were housed in groups, and therefore a precise determination of the amount of CDP-choline ingested per rat was not available.

\section{Rearing conditions}

All rats were housed in the same rack in plastic cages $(52 \times 32 \times$ $20 \mathrm{~cm}$ high) with wire lids. Bedding and water were regularly changed, and animals were weighed each week, at which time general health assessments were made. Animals had ad libitum access to chow and water. EC rats were housed in groups of 3-4 animals. Plastic toys (blocks, balls, cylinders, etc.) placed in the EC cages were rotated between groups weekly; new toys were introduced monthly. EC rats were taken to a 'playroom' $(12 \times 6$ $\mathrm{ft}$; containing filing cabinets, desks, chairs, boxes and toys) every other day for $30-45 \mathrm{~min}$. The IC rats were housed individually, without toys, and handled every other day to acclimatize the animals to experimenter handling and in order to alleviate fear and anxiety in subsequent behavioral training procedures. To avoid the typical weight gain caused by impoverished conditions (relative to enriched rats), rats were allowed to exercise three times per week for $15 \mathrm{~min}$ in an empty $4 \times 6 \mathrm{ft}$ room with only an experimenter present.

\section{Water maze apparatus}

The water maze was a black circular tank $6 \mathrm{ft}(1.83 \mathrm{~m})$ in diameter and $1.5 \mathrm{ft}(0.55 \mathrm{~m})$ in height, filled with water $\left(25^{\circ} \mathrm{C} \pm 2^{\circ} \mathrm{C}\right)$ to a depth of $20 \mathrm{~cm}$ and located in a room containing several extramaze cues. Four starting positions (north, south, east, west) were spaced around its perimeter, dividing the pool into four equal quadrants. The rectangular clear Plexiglas escape platform used for the spatial task $(11 \times 14 \times 19 \mathrm{~cm})$ was submerged at a depth of $1 \mathrm{~cm}$. For the cued version of the water maze, a white rubber ball $(8 \mathrm{~cm}$ in diameter) attached to the top of the submerged platform protruded above the water surface. The platform could be used as a step to mount the ball to escape the water.

\section{Behavioral procedures}

The following experiments were carried out in accordance with The National Institutes of Health Guide for the Care and Use of Laboratory Animals. All efforts were made to minimize animal suffering and to reduce the number of animals used. Behavioral testing was carried out in a blind manner.

\section{Spatial water maze (hidden platform task)}

Rats received a 4-d training session consisting of four trials (i.e., swims) per day in the spatial water maze task. On each trial the animal was placed into the tank facing the wall at one of four designated start points $(\mathrm{N}, \mathrm{S}, \mathrm{E}$, and $\mathrm{W})$ and allowed to escape onto the hidden platform. The submerged platform was located in the same quadrant (quadrant N) on every trial for all $4 \mathrm{~d}$. A different starting point was used on each trial such that each starting point was used once each day. If an animal did not escape within $90 \mathrm{sec}$, it was manually guided to the escape platform by the experimenter. After mounting the platform, rats remained on the platform for $20 \mathrm{sec}$. Following each trial, animals were removed from the maze and placed in a holding cage for a 30-sec intertrial interval (ITI). The latency to mount the escape platform was recorded and used as a measure of task acquisition.

Twenty-four hours after the final training day (i.e., day 5), rats were given a 60 -sec probe test (where the platform was removed from the pool). During the probe trials, the time spent in each of the four quadrants was recorded and analyzed. 


\section{Cued water maze (hidden platform task)}

One week after completion of the 4-day/4 trials per day spatial training task, rats received three training sessions consisting of four trials (i.e., swims) per day in the cued water maze task. On each trial the animal was placed into the tank facing the wall at one of four designated start points (N, S, E, and W) and allowed to escape onto the visibly cued platform. A different starting point was used on each trial such that each starting point was used each day. If an animal did not escape within 90 sec, it was manually guided to the escape platform by the experimenter. After mounting the platform, rats remained on the platform for $20 \mathrm{sec}$. Following each trial, animals were removed from the maze and placed in a holding cage for a 30-sec ITI. The latency to mount the escape platform was recorded and used as a measure of task acquisition. The visible escape platform was placed in a different quadrant on each trial such that each of the four quadrants contained the escape platform once each day.

\section{Data analysis}

Data are expressed as means \pm SEMs. $P$-values $<0.05$ were considered statistically significant. Experimental groups were compared using one-way analysis of variance (ANOVA) with repeated measures, followed by Scheffe's post-hoc test to compare between groups if overall significance was found by ANOVA.

\section{Acknowledgments}

This study was supported in part by grants from The National Institutes of Mental Health (Grant No. 5-RO1 MH28783-24) and The Center for Brain Sciences and Metabolism Charitable Trust. We thank Dr. Charles Benton of Harlan Teklad for his assistance with the diets.

\section{References}

Albert, M. 1993. Neuropsychological and neurophysiological changes in healthy adult humans across the age range. Neurobiol. Aging 14: $623-625$.

Barnes, C.A., Nadel, L., and Honig, W.K. 1980. Spatial memory deficits in senescent rats. Can. J. Psych. 34: 29-39.

Cohen, E.L. and Wurtman, R.J. 1976. Brain acetylcholine: Control by dietary choline. Science 191: 561-562.

Craik, F.L. 1990. Changes in memory with normal aging: A functional view. Adv. Neurol. 51: 201-205.

Duffy, S.N., Craddock, K.J., Abel, T., and Nguyen, P.V. 2001. Environmental enrichment modifies the PKA-dependence of hippocampal LTP and improves hippocampal-dependent memory. Learn. Mem. 8: 26-34.

Gage, F.H., Chen, K.S., Buzsaki, G., and Armstrong, D. 1988. Experimental approaches to age-related cognitive impairments. Neurobiol. Aging 9: 645-655.

Gallagher, M. and Pelleymounter, M.A. 1988. Spatial learning deficits in old rats: A model for memory decline in the aged. Neurobiol. Aging 9: $81-88$.
Gallagher, M., Burwell, R., and Burchinal, M. 1993. Severity of spatial learning impairment in aging: Development of a learning index for performance in the Morris water maze. Behav. Neurosci. 107: $618-626$

Hebb, D.O. 1949. The Organization of Behavior. Wiley, New York.

Hirsh, M.J., Growdon, J.H., and Wurtman, R.J. 1978. Relations between dietary choline or lecithin intake, serum choline levels, and various metabolic indices. Metabolism 27: 953-960.

Holscher, C., Caneveri, L., Richter-Levin, G. 1995. Inhibitors of PLA and NO synthase cooperate in producing amnesia of a spatial task. Neuroreport 6: 730-732.

Juraska, J.M.H. and Muller, J. 1984. Differential rearing experience, gender, and radial maze performance. Dev. Psychobiol. 17: 209-215.

Kempermann, G., Kuhn, H.G., and Gage, F.H. 1997. More hippocampal neurons in adult mice living in enriched environment. Nature 386: 493-495.

Lopez-Coviella, G., Agut, J., and Wurtman, R.J. 1987. Metabolism of cytidine (5')-diphopshocholine (CDP-choline) following oral and intravenous administration to the human and the rat. Neurochem. Internatl. 11: 293-297.

Lopez-Coviella, G., Agut, J., Ortiz, J.A., and Wurtman, R.J. 1992. Effects of orally administered cytidine 5 '-diphosphate choline on brain phospholipid content. J. Nutritional. Biochem. 3: 313-315.

Moriyama, T., Uezu, K., Matsumoto, Y., Chung, S.Y., Uezu, E., Miyagi, S., Uza, M., Masuda, Y., Kokubu, T., Tanaka, T., et al. 1996. Effects of dietary phosphatidylcholine on memory deficient mice with low brain acetylcholine. Life Sci. 58: PL111-PL118.

Morris, R.G.M., Garrud, P., Rawlins, J.N.P., and O'Keefe, J. 1982. Place navigation impaired in rats with hippocampal lesions. Nature 297: 681-683.

Packard, M.G. and Teather, L.A. 1997. Double dissociation of hippocampal and dorsal-striatal memory systems by posttraining injections of 2-amino-5-phosphopentanoic acid. Behav. Neurosci. 111: $543-551$.

. 1998. Amygdala modulation of multiple memory systems: Hippocampus and caudate-putamen. Neurobiol. Learn. Mem. 69: 163-203.

Packard, M.G., Hirsh, R., and White, N.M. 1989. Differential effects of fornix and caudate nucleus lesions on two radial maze tasks: Evidence for multiple memory systems. J. Neurosci. 9: 14651472.

Rapp, P.R., Rosenberg, R.A., and Gallagher, M. 1987. An evaluation of spatial information processing in aged rats. Behav. Neurosci. 101: $3-12$.

Teather L.A. and Wurtman, R.J. 2003. Dietary cytidine (5')-diphosphocholine supplementation protects against development of memory deficits in aging rats. Prog. Neuropsychopharmacol. Biol. Psychiatry 27: 711-717.

Teather, L.A., Packard, M.G., and Bazan, N.G. 1998. Effects of post-training intra-hippocampal injections of platelet-activating factor and PAF antagonists on memory. Neurobiol. Learn. Mem. 70: $349-363$.

. 2002. Post-training cyclooxygenase-2 (COX-2) inhibition impairs memory consolidation. Learn. Mem. 9: 41-47.

Received July 9, 2003; accepted in revised form November 3, 2004. 


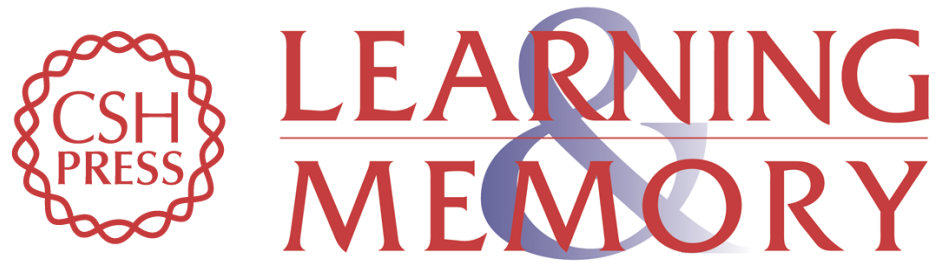

\section{Dietary CDP-choline supplementation prevents memory impairment caused by impoverished environmental conditions in rats}

Lisa A. Teather and Richard J. Wurtman

Learn. Mem. 2005, 12:

Access the most recent version at doi:10.1101/lm.83905

References This article cites 21 articles, 4 of which can be accessed free at: http://learnmem.cshlp.org/content/12/1/39.full.html\#ref-list-1

License

Email Alerting Receive free email alerts when new articles cite this article - sign up in the box at the Service top right corner of the article or click here. 